\title{
BMJ Open Portrait of trauma care in Quebec's rural emergency departments and identification of priority intervention needs to improve the quality of care: a study protocol
}

\author{
Richard Fleet, ${ }^{1,2}$ Fatoumata Korika Tounkara, ${ }^{2}$ Mathieu Ouimet, ${ }^{3}$ Gilles Dupuis, ${ }^{4}$ \\ Julien Poitras, ${ }^{1}$ Alain Tanguay, ${ }^{2}$ Jean Paul Fortin, ${ }^{5}$ Jean-Guy Trottier, ${ }^{6}$ \\ Jean Ouellet, ${ }^{1}$ Gilles Lortie, ${ }^{1}$ Jeff Plant, ${ }^{7}$ Judy Morris, ${ }^{8}$ Jean Marc Chauny, ${ }^{8}$ \\ François Lauzier, ${ }^{9}$ France Légaré ${ }^{10}$
}

To cite: Fleet $\mathrm{R}$, Tounkara FK, Ouimet $\mathrm{M}$, et al. Portrait of trauma care in Quebec's rural emergency departments and identification of priority intervention needs to improve the quality of care: a study protocol. BMJ Open 2016;6: e010900. doi:10.1136/ bmjopen-2015-010900

- Prepublication history for this paper is available online. To view these files please visit the journal online (http://dx.doi.org/10.1136/ bmjopen-2015-010900).

Received 17 December 2015 Revised 17 February 2016 Accepted 29 February 2016

CrossMark

For numbered affiliations see end of article.

Correspondence to Dr Richard Fleet; rfleet@videotron.ca

\section{ABSTRACT}

Introduction: Trauma remains the primary cause of death in individuals under 40 years of age in Canada. In Quebec, the Trauma Care Continuum (TCC) has been demonstrated to be effective in decreasing the mortality rate among trauma victims. Although rural citizens are at greater risk for trauma and trauma death, no empirical data concerning the effectiveness of the TCC for the rural population in Quebec are available. The emergency departments (EDs) are important safety nets for rural citizens. However, our data indicate that access to diagnostic support services, such as intensive care units and CT is limited in rural areas. The objectives are to (1) draw a portrait of trauma services in rural EDs; (2) explore geographical variations in trauma care in Quebec; (3) identify adaptable factors that could reduce variation; and (4) establish consensus solutions for improving the quality of care.

Methods and analysis: The study will take place from November 2015 to November 2018. A mixed methodology (qualitative and quantitative) will be used. We will include data (2009-2013) from all trauma victims treated in the 26 rural EDs and tertiary/ secondary care centres in Quebec. To meet objectives 1 and 2, data will be gathered from the Ministry's Database of the Quebec Trauma Registry Information System. For objectives 3 and 4 , the project will use the Delphi method to develop consensus solutions for improving the quality of trauma care in rural areas. Data will be analysed using a Poisson regression to compare mortality rate during hospital stay or death on ED arrival (objectives 1 and 2). Average scores and $95 \% \mathrm{Cl}$ will be calculated for the Delphi questionnaire (objectives 3 and 4).

Ethics and dissemination: This protocol has been approved by CSSS Alphonse-Desjardins research ethics committee (Project MP-HDL-2016-003). The results will be published in peer-reviewed journals.

\section{Strengths and limitations of this study}

This is the first study examining trauma care in rural Canada, in particular, the province of Quebec.

- In the short term, our results will generate empirical data that will draw a portrait of trauma cases treated in rural emergency departments (EDs); document inequalities and the impact of variability in rural ED and urban trauma care.

- The study will allow us to evaluate the effectiveness of the trauma care continuum for the rural population in Quebec.

- Our results will justify implementation of a new strategy promoting rapid care for rural trauma victims.

- We expect that current clinical databases may not capture all trauma victims in rural areas, particularly those who are treated in a nondesignated trauma centre.

\section{INTRODUCTION}

Since the 1992 implementation of an organised trauma system in the province of Quebec: The Trauma Care Continuum (TCC), mortality rate for victims of a severe trauma in Quebec has decreased from 52\% to $8.6 \% .^{1}$ However, the TCC is organised in such a way that certain areas of the province, mainly rural, remain at a significant distance from regional/provincial trauma centres. Approximately one half of the rural emergency departments (EDs) in Quebec are over $300 \mathrm{~km}$ from a tertiary or secondary traumatology centre, and our data indicate that access to diagnostic support services, such as intensive care units (ICU) and CT scanner is often limited in rural areas. ${ }^{2-5}$ 
Furthermore, poor road conditions and an elevated rate of high-risk activities (eg, farming) increase the incidence of trauma in rural areas. ${ }^{67}$

No specific data are available on the effectiveness of the TCC for the rural population in Quebec. Data from elsewhere in Canada suggest that, for similar injuries, the mortality rate is twice as high for rural trauma victims than for urban victims. ${ }^{8}$ This finding is hypothesised to be attributable to rural medical personnel's limited exposure to trauma, delays in reporting trauma, limited access to trauma centres, lenghty ambulance transports and limited in-hospital resources. ${ }^{8-12}$

In Quebec, rural EDs receive over 500000 visits per year, and a significant proportion of consultations are trauma related. ${ }^{3}$ Ultimately, the effectiveness of the TCC cannot be ascertained until rural and urban patients in Quebec are receiving a same quality treatment for traumatic injuries. Identification of adaptable factors that explain variation across establishments constitutes a further critical step in developing potential organisational solutions. This project is designed to establish the status of treatment for traumatic injuries in rural and urban Quebec, and to evaluate the contribution of rural EDs toward optimising the TCC.

\section{Trauma and trauma care practices}

On a global scale, trauma is a major public health issue. ${ }^{13} 14$ In 2004, approximately 1 million individuals consulted EDs subsequent to a traumatic injury, and over 200000 were hospitalised. ${ }^{15}$ Despite improvement in trauma care and services, trauma remains the primary cause of death in individuals under 40 years of age in Canada. ${ }^{16}$

Trauma care has considerably improved over the past 40 years, with the establishment of new trauma networks, including hospitals (trauma centres) that offer complete access to diagnostic and technological services, and to ultraspecialised medical and surgical care. ${ }^{17}{ }^{18}$ Although the overall efficacy of trauma networks has been demonstrated, experts agree that existing systems are not conducive to effective care in rural or isolated areas, particularly in Canada. ${ }^{17-21}$ In Canada, over 7 million citizens $(22.5 \%$ of the population), primarily rural, live over an hour's drive away from a trauma centre. ${ }^{17}$ The 'Golden Hour' principle proposes that trauma victims with rapid access to a trauma centre have a greater chance of survival. ${ }^{22}$

\section{Shortcomings in existing studies of rural trauma care}

Although 19\% of Quebeckers and Canadians live in rural areas, ${ }^{23}$ and rural citizens have an elevated rate of mortality subsequent to trauma, ${ }^{21}{ }^{24}$ there remains a lack of empirical data on rural trauma care. The majority of published studies on rural trauma care were conducted in the USA, and the results are not necessarily applicable in Canada. The two countries differ significantly in terms of population density, ${ }^{17}$ healthcare system, prehospital care systems, access to human and technological resources in hospitals ${ }^{18}$ and training of emergency physicians. ${ }^{25}$

Since 2010, the Research Chair in Emergency Medicine at Laval University/Hotel-Dieu de Lévis has been collaborating with interdisciplinary and interuniversity researchers to improve the quality of care in rural EDs in Canada. The team recently completed two large-scale studies describing the services offered in the 26 rural EDs in Quebec and 325 rural EDs in Canada. $^{2} 3552627$ The results indicate that rural EDs have limited access to certain services, as well as to diagnostic equipment (ICU, CT scanner, etc) and medical specialists (general surgeons, intensivists, anaesthesiologists, etc). ${ }^{2} 3527$ Our results suggest that many professionals report the need for supplementary training in emergency medicine/traumatology, and that many doctors practising in rural areas contemplate leaving rural regions in search of a less demanding practice. ${ }^{28}$ The logical next step in our research programme is to draw a detailed portrait of trauma care in rural EDs in Quebec and explore the feasibility of the existing proposed solutions; next, further potential solutions will be developed once the present situation and current needs have been established.

\section{Solutions for improving trauma care in rural areas}

Given unavoidable distances between trauma centres and the urgent nature of trauma treatment, improving rural traumatology requires quality trauma care in rural EDs, and optimisation of emergency prehospital services (PHS).

The waiting period creates delays in treatment and negatively influences clinical prognosis. ${ }^{29}$ The necessity of extended clinical investigation and delays in organising transfers has been challenged, and some researchers have rightfully suggested that trauma victims would benefit from direct transportation from trauma site to trauma centre. Experts from the Quebec Institute for Excellence in Health and Social Services (INESSS) are exploring the possibility of promoting direct transfer of trauma patients to tertiary or secondary care centres, avoiding small rural EDs altogether. ${ }^{30}$ INESSS recommends a maximum of $45 \mathrm{~min}$ (or $75 \mathrm{~km}$ ) for direct transfers. $^{30}$ According to our data, $96 \%$ and $88 \%$ of rural EDs in Quebec are over $75 \mathrm{~km}$ from tertiary and secondary trauma care centres, respectively. Given this finding, the possibility of bypassing rural EDs warrants further exploration.

\section{Telemedicine support for interestablishment transfers}

Safe, rapid transfer to trauma centres is essential for victims of serious injuries who are initially stabilised in rural EDs. The practice of interestablishment transfer from rural EDs to tertiary care centres in Quebec has not been studied. A clear portrait of the status quo will highlight any necessary adaptations to this critical link in the trauma care chain. For example, the limited medical personnel in rural areas creates a challenge for the 
proposed solution of medical escorts during transfers. A telemedicine programme in the receiving trauma centre could constitute a viable solution for supporting ambulance and nursing staff during transfers. ${ }^{31}$

\section{Improving trauma care in rural EDs}

Several solutions have been proposed for improving care for trauma victims in rural EDs. They include re-evaluating resource attribution, use of new technology, and implementation of novel training programmes for personnel. The lack of resources in rural areas necessitates an elevated rate of interestablishment transfers ${ }^{32} 33$ creating significant strain on PHS and negatively impacting care. Variables for consideration in service attribution decisions include number of consultations in the given ED, severity of medical and trauma cases, distance from a trauma centre, current number of required transfers, and the needs of citizens and professionals in the rural areas.

\section{Use of new technology}

Use of new technology such as Point-Of-Care Ultrasound (POCUS) can significantly improve the quality of trauma care in rural regions. ${ }^{34}$ Given limited access to advanced imaging technology (CT scanner and MRI) in rural EDs, POCUS could constitute one solution for improving quality of care. ${ }^{34}$ POCUS can be used by nonradiologists, and facilitates rapid and safe evaluation of potentially fatal conditions, as well as several non-fatal medical and obstetrical conditions.

\section{OBJECTIVES}

This project is designed to improve the quality of trauma care in rural EDs in Quebec. The primary objectives of the project are to:

- Draw a portrait of trauma services in rural EDs.

- Explore geographical variations in trauma care in Quebec.

- Identify adaptable factors that could reduce variation across establishments.

- Establish consensus solutions for improving the quality of care.

\section{METHODS AND ANALYSIS}

The study will take place from April 2016 to April 2019. A mixed methodology will be used (qualitative and quantitative). The project will be a multicentre study in rural EDs and tertiary and secondary trauma care centres in Quebec. In a previously conducted pilot study, rural EDs were identified using the Health Canada Establishment Guide. $^{26}$ They offer 24/7 medical care, have hospital beds, and are situated in rural and small towns as per the Statistics Canada definition (population $>10000$ and population density $<400 / \mathrm{km}^{2}$ or population $<10000$ and population density $>400 / \mathrm{km}^{2}$ or population $<10000$ and population density $<400 / \mathrm{km}^{2}$ ). Rural communities in Quebec were identified using Statistics
Canada criteria. The sample will be composed of data from all trauma victims treated in the 26 rural EDs in Quebec (including primary care centres), and from all victims treated in the 3 tertiary care centres for adults, 3 paediatric tertiary care centres, 4 regional secondary care centres and 22 secondary care centres.

Phase 1: generate a portrait of trauma care in rural EDs and explore geographical variations in trauma services in Quebec (objectives 1 and 2)

\section{Data sources}

To meet objectives 1 and 2, data from 2009 to 2013 will be collected from the Ministry's Database of the Quebec Trauma Registry Information System (BDM-SIRTQ), and from data collected in our previous project. ${ }^{26}$ The BDM-SIRTQ contains all information on victims of a traumatic event causing injury, victims who died on arrival at the ED or during ED stay, and victims who were hospitalised in a designated trauma centre in Quebec. ${ }^{35}$ The data collected in our project on Quebec's rural EDs includes information on the hospital, the ED, prehospital emergency services and interestablishment transfers.

\section{Portrait of trauma in rural EDs (objective 1)}

Data from patients treated in rural as well as well as those treated in tertiary or secondary care centres will be used to describe patient and service characteristics. The data will focus on (1) traumatic event data (eg, age and gender, injured area of the body, date and time of the traumatic event, cause of trauma, emergency service response code (eg, dead on arrival, death during transportation, living), the prehospital trauma index score (PTI), the Injury Severity Score (ISS), etc); (2) injury data (eg, injury code, Abbreviated Injury Scale (AIS) score, and diagnosis code from 1 to 5 (code indicating the traumatic injury according to the International Classification of Diseases); (3) Hospital stay data (eg, sequential trauma score, stay number, type of stay, source of trauma, etc); (4) treatment data (eg, treatment code (this field specifies the code of medical interventions or medical treatments that are performed medically and/or surgically in the emergency department and/or a facility admission), diagnosis and surgery during ED stay, etc).

\section{Exploration of geographical variation in trauma care in Quebec (objective 2)}

The second objective of the project is to explore geographical variation in trauma care in Quebec and to identify inequalities between rural and urban areas. BDM-SIRTQ data will be combined with data collected in our project on Quebec's rural EDs.

We will evaluate the variation in services (EDs resources, staff compliment); we will compare the mortality rate among patients treated in rural EDs versus those treated in urban centres. Data will focus on the number of deaths during ED stay, access to specialists, 
intensive care, medical imaging, and so on. To explore geographical variation in trauma care in Quebec, we will compare the prehospital mortality among rural versus urban patients. We will capture data on the number of prehospital deaths, mode of patient's transport (eg, air plan, ambulance, helicopter, etc), the prehospital time, and so on.

Phase 2: identify adaptable factors that could reduce variations across geographical regions and establish a consensus among partners regarding solutions for improving the quality of trauma care (objectives 3 and 4) Delphi study

This phase of the project will use the Delphi method to develop consensus solutions for improving the quality of trauma care in rural regions. The Delphi method is an effective method for reaching consensus on a specific theme. ${ }^{36}{ }^{37}$ It was chosen because it 'is the method of choice in emergency medicine/trauma, and has been demonstrated to be effective for establishing expert consensus. ${ }^{38} 39$ The method is based on a structured process involving the development of successive iterations of a questionnaire and feedback on the responses received for each iteration. Number of iterations depends on the rapidity with which consensus is reached. The threshold for consensus in the present study will be $80 \%$, as established in the literature in this area. ${ }^{40}$

\section{Delphi participant recruitment and inclusion criteria}

The first step in the Delphi method is identification and recruitment of experts interested in trauma care in rural regions, and who are prepared to commit to the process. The principle of international (deliberate) sampling will be used to select participants. ${ }^{41}{ }^{42}$ Participant selection is designed to include the widest possible range of experts and to obtain the broadest scope of information. ${ }^{43-45}$ Experts will be chosen based on contribution to the field of traumatology, with the objective of creating a team with a wide variation in expertise and experience. The experts included in the study will include ED physicians, nurses, neurosurgeons, general surgeons and intensivists in tertiary and secondary trauma care centres, as well as physicians practising in rural EDs. Specialists in PHS, telemedicine, simulation-based learning and POCUS will be invited to participate. Representatives from the Quebec association of ED Physicians, the Canadian Association of Emergency Physicians, and the College of Physicians of Quebec will be invited to participate in this step of the study.

\section{Delphi data collection}

Given constraints on participants' time, the present study will collect data using LimeSurvey, an online programme recommended by the Laval University ethics committee for research with human subjects. To avoid loss of motivation, participants will be given a limit of 2 weeks to complete the questionnaires.

\section{First iteration: Questionnaire 1}

The first questionnaire will incorporate the key elements that emerged from our analyses for Objectives 1 and 2; data from consultations with professionals in rural EDs; data from existing North American literature on improving trauma care in rural regions; reports produced by INESSS; data from consultations with specialists in the Quebec trauma care network; and data from consultations with specialists in PHS experts, telemedicine, POCUS, and simulation-based learning. The questionnaire will be subject to a pilot test to verify content and format. The first questionnaire will consist of open-ended questions based on our existing knowledge. Four primary themes will be addressed: improving PHS (including optimising interestablishment transfers), improving access to ED services, use and implementation of new technology, and physician continuing education. Participants will be asked to list potential solutions for improving trauma care in rural regions, and to include brief written comments for each proposed solution.

\section{Second iteration: Questionnaire 2}

The responses from the first version of the questionnaire will be compiled and analysed. Statistics professionals will use Nvivo software to conduct qualitative analysis and to organise proposed solutions by theme. The themes are expected to be consistent with the four primary themes described above, and new themes may also emerge. A second online questionnaire will be developed based on the themes from the first questionnaire. The second questionnaire will be sent to participants; they will be asked to comment on each proposed solution, and to use a 5-point Likert scale to identify the most relevant of the proposed solutions for the established priorities.

\section{Third iteration: Questionnaire 3}

The responses from the second questionnaire will be compiled and analysed to identify convergent and divergent points for each theme. A third questionnaire will be developed and sent to participants. In this round, each participant will receive the priority score for each solution averaged across all participants, as well as a reminder of his or her initial position. Participants will cast a final vote on the retained solutions, judging each solution as 'nonrelevant', 'somewhat relevant', 'relevant' or 'very relevant'. Although the Delphi method is usually complete after a third iteration, the present study will continue to collect data in the event of non-consensus after the third round.

\section{Statistical analyses}

The statistical analyses will be conducted by the statistics consultation service at Laval University. A professional on qualitative data will conduct the qualitative analyses. Descriptive statistics (mean, median, percentages, according to distribution of variables) will be presented to meet the objective 1 .

To meet objective 2, a Poisson regression analysis will compare the rate of mortality during hospital stay 
between 2009 and 2013 for trauma victims treated in rural EDs, and trauma victims treated in trauma centres. The dependent variable will be the number of deaths during ED stay, with total number of trauma victims as the offset. Trauma victims treated in secondary and tertiary care centres subsequent to transfer from a rural region will constitute the 'rural region' group. Potential explanatory variables will include average case severity, average patient age, patient gender, availability of local resources (specialists, intensive care, medical imaging), characteristics of the rural ED (visits per year, number of stretchers, number of intensive care beds), average stay at ICU, average duration of hospital stay and average duration of stay in rehabilitation. Selection of explanatory variables was based on the existing literature in this area. ${ }^{7} 104647$ A propensity score will be calculated to control for confounding factors. A propensity score promotes greater statistical precision by significantly reducing sources of bias. ${ }^{48}$

Where death on ED arrival is concerned, we will closely explore variables associated with the links in the chain of intervention in prehospital emergency care. A Poisson regression model will be used to compare rate of death on ED arrival. Explanatory variables will include date and time of the trauma, transport time, and distance between rural ED and trauma centre. The relationship between these variables and prehospital mortality has been demonstrated elsewhere. ${ }^{49}$

The data collected as part of the phase 2 will be compiled and analysed. Average scores, as well as average 95\% CI, will be calculated for each iteration of the questionnaire. IQR will be used as a statistical dispersion measure to evaluate the strength of the consensus. ${ }^{36}$

\section{ETHICS AND DISSEMINATION}

This project required ethics evaluation through a multicentre study mechanism described below. In the new law of the province of Québec, a study that is conducted in several centres must be evaluated by a main research centre ethics committee (main REC), which is normally the REC that belongs to the research centre where the project is initiated. Therefore, the project is not evaluated by the REC of each study site. The project has been approved by CSSS Alphonse-Desjardins research ethics committee (Project MP-HDL-2016-003). The results will be published in peer-reviewed scientific journals.

\section{DISCUSSION AND EXPECTED RESULTS}

The TCC was established to improve the quality of trauma care for Quebec citizens. However, the effectiveness of the system for the province's rural population is unknown. Our project constitutes a significant step in a novel and innovative research programme on rural trauma care. The research team includes experts in rural medicine, emergency medicine, traumatology, healthcare systems management and knowledge transfer.
Victims of severe trauma require rapid care and transfer to an appropriate treatment centre within a reasonable delay. ${ }^{10} 47$ The process entails considerable costs, and exposes trauma victims to the risks inherent in interestablishment transfers. Our project will present a detailed portrait of trauma cases treated in rural EDs, and of the quality of care offered. It will improve our understanding of the use of EDs by trauma victims in rural regions. Increased information about the rationales for transfers will help us determine whether or not rural EDs, and the existing trauma care system, respond adequately to the needs of the rural population. In illuminating this issue, our project will have an immediate and significant impact on rural trauma victims.

We expect to discover significant disparities between the quality of care in rural and urban regions. We further expect that our results will justify implementation of a new strategy promoting rapid care for rural trauma victims. The potential solutions generated by this project will address the needs of citizens, professionals and policymakers in Quebec's rural areas and will constitute a critical step in improving trauma care for this vulnerable population. Finally, we expect that the relationships we developed with partners outside of Quebec over the course of our prior study of rural EDs in Canada will inspire future pan-Canadian and international initiatives.

\section{Author affiliations}

${ }^{1}$ Department of Family and Emergency Medicine, Université Laval, Québec, Quebec, Canada

${ }^{2}$ Research Chair in Emergency Medicine, Univerité Laval - CHAU Hôtel-Dieu de Lévis, Lévis, Quebec, Canada

${ }^{3}$ Department of Political Science, Université Laval, Québec, Quebec, Canada ${ }^{4}$ Département de Psychologie, Université du Québec à Montréal, Montréal, Quebec, Canada

${ }^{5}$ Département de Médecine Sociale et Préventive, Université Laval, Québec, Quebec, Canada

${ }^{6}$ Centre de Santé et de Services Sociaux de l'Hématite, Fermont, Quebec,

Canada

${ }^{7}$ Department of Emergency Medicine, University of British Columbia, Vancouver, British Columbia, Canada

${ }^{8}$ Hôpital du Sacré-Cœur de Montréal, Université de Montréal, Montréal, Quebec, Canada

${ }^{9} \mathrm{CHU}$ de Québec and Université Laval, Research center, Quebec, Quebec, Canada

${ }^{10}$ Department of Family Medicine and Emergency Medicine, Knowledge Transfer and Health Technology Assessment of the CHUQ Research Centre (CRCHUQ), Unité de Recherche Évaluative, Université Laval, Québec, Quebec, Canada

Acknowledgements The authors wish to thank the rural emergency staff of the province of Québec for participating in our previous study, as well of the government agencies ('Régie de l'assurance maladie du Québec') who collaborated in providing the data. They would also like to thank Denise Trudel, Alan Drummond (Canadian Association of Emergency Physicians), and Dr Bernard Mathieu (Quebec Association of Emergency Medicine). Finally, they wish to thank the "Direction des soins critiques et urgents Ministère de la Santé et des Services sociaux" for their collaboration.

Contributors RF was responsible for the original idea, literature review and study design. He drafted the initial manuscript and its revised versions. FKT contributed significantly to the manuscript drafting and preparation, revision and formatting the manuscript. MO, GD, JP, JPF, FL, J-GT, JO, GL, AT and JP have contributed to various aspects of the study design with input relating to 
their specific expertise in the field. All authors read and approved the final manuscript.

Funding The research project is supported from the "Fonds de Recherche du Québec-Santé (FRQS)" 31033.

Competing interests None declared.

Ethics approval CSSS Alphonse-Desjardins research ethics committee.

Provenance and peer review Not commissioned; externally peer reviewed.

Open Access This is an Open Access article distributed in accordance with the Creative Commons Attribution Non Commercial (CC BY-NC 4.0) license, which permits others to distribute, remix, adapt, build upon this work noncommercially, and license their derivative works on different terms, provided the original work is properly cited and the use is non-commercial. See: http:// creativecommons.org/licenses/by-nc/4.0/

\section{REFERENCES}

1. Institut national d'excellence en santé et en services sociaux (INESSS). Les caractéristiques, l'historique et l'implantation du continuum de services en traumatologie du Québec (1991-2012). Montréal, QC: INESSS, 2012:85.

2. Fleet R, Archambault P, Poitras J, Villa J, eds. Limited access to services in Canada rural emergency departments: pilot study. CAEP 2012 Annual Conference; Niagara Falls, Canada: CJEM, 2012.

3. Fleet R, Poitras J, Archambault P, et al. Portrait of rural emergency departments in Québec and utilization of the provincial emergency department management Guide: cross sectional survey. BMC Health Services Research 2015

4. Fleet R, Audette LD, Marcoux J, et al. Comparison of access to services in rural emergency departments in Quebec and British Columbia. CJEM 2014;16:437-48.

5. Fleet R, Poitras J, Maltais-Giguere J, et al. A descriptive study of access to services in a random sample of Canadian rural emergency departments. BMJ Open 2013;3:e003876.

6. Merchant JA, Reynolds S, Zwerling C. Work in agriculture. In: McDonald JC, ed. Epidemiology of work related diseases. London, England: BMJ Publishing Group, 1995:267-92.

7. Peek-Asa C, Zwerling C, Stallones L. Acute traumatic injuries in rural populations. Am J Public Health 2004;94:1689-93.

8. Fatovich DM, Jacobs IG. The relationship between remoteness and trauma deaths in Western Australia. J Trauma 2009;67:910-14.

9. Artuso CE. Rural trauma challenges in Alaska. Crit Care Nurs Clin North Am 2012;24:593-600.

10. Gomez D, Berube M, Xiong W, et al. Identifying targets for potential interventions to reduce rural trauma deaths: a population-based analysis. J Trauma 2010;69:633-9.

11. Harrington DT, Connolly M, Biffl WL, et al. Transfer times to definitive care facilities are too long: a consequence of an immature trauma system. Ann Surg 2005;241:961-6; discussion 6-8.

12. Weiss S, Fullerton L, Oglesbee S, et al. Does ambulance response time influence patient condition among patients with specific medical and trauma emergencies? South Med J 2013;106:230-5.

13. Trunkey D. Initial treatment of patients with extensive trauma. $N$ Engl J Med 1991;324:1259-63.

14. Centers for Disease Control and Prevention, National Center for Injury Prevention and Control. http://www.nationaltraumainstitute.org/ home/trauma_statistics.html (accessed 18 Nov 2014).

15. SMARTRISK. The economic burden of injury in Canada. http://www. parachutecanada.org/research/item/economic-burden-of-injuryreports (accessed 10 Nov 2014).

16. Statistics Canada. Leading causes of death, by sex, Canada. http:// www.statcan.gc.ca/tables-tableaux/sum-som/101/cst01/hlth36a-eng. htm (accessed Sep 2015).

17. Hameed S, Schuurman N, Razek T, et al. Access to trauma systems in Canada. J Trauma 2010;69:1350-61.

18. Zakrison T, Ball CG, Kirkpatrick AW. Trauma in Canada: a spirit of equity \& collaboration. World J Surg 2013;37:2086-93.

19. Mullins RJ, Hedges JR, Rowland DJ, et al. Survival of seriously injured patients first treated in rural hospitals. J Trauma 2002;52:1019-29.

20. Rogers FB, Osler TM, Shackford SR, et al. Study of the outcome of patients transferred to a level I hospital after stabilization at an outlying hospital in a rural setting. J Trauma 1999;46:328-33.

21. Simons R, Brasher $P$, Taulu $T$, et al. A population-based analysis of injury-related deaths and access to trauma care in rural-remote Northwest British Columbia. J Trauma 2010;69:11-19.
22. Nathens AB, Brunet FP, Maier RV. Development of trauma systems and effect on outcomes after injury. Lancet 2004;363:1794-801.

23. Statistics Canada. Population, urban and rural, by province and territory. http://www.statcan.gc.ca/tables-tableaux/sum-som/l01/ cst01/demo62f-eng.htm (accessed 18 Nov 2014).

24. Bell N, Simons RK, Lakha N, et al. Are we failing our rural communities? Motor vehicle injury in British Columbia, Canada, 2001-2007. Injury 2012;43:1888-91.

25. Sklar DP, Handel DA, Hoekstra J, et al. The future of emergency medicine: an evolutionary perspective. Acad Med 2010;85:490-5.

26. Fleet R, Archambault P, Legare F, et al. Portrait of rural emergency departments in Quebec and utilisation of the Quebec Emergency Department Management Guide: a study protocol. BMJ Open 2013;3:e002961.

27. Fleet R, Pelletier C, Marcoux J, et al. Differences in access to services in rural emergency departments of Quebec and Ontario. PLOS ONE 2015;10:e0123746.

28. Drouin MA, Fleet R, Poitras J, et al. The Quebec rural emergency department project: a cross-sectional study of a potential two-pronged strategy in the knowledge transfer process. PLoS ONE 2015;10:e0120523.

29. Humber N, Frecker T. Rural surgery in British Columbia: is there anybody out there? Can J Surg 2008;51:179-84.

30. Institut national d'excellence en santé et en services sociaux (INESSS). Avis sur les critères de triage préhospitalier en traumatologie. Rapport rédigé par André Lavoie en collaboration avec Gilles Bourgeois et Jean Lapointe. ETMIS 2013;9:1-46.

31. Charash WE, Caputo MP, Clark $\mathrm{H}$, et al. Telemedicine to a moving ambulance improves outcome after trauma in simulated patients. J Trauma 2011;71:49-54; discussion 5.

32. Fleet R, Plant J, Ness R, et al. Patient advocacy by rural emergency physicians after major service cuts: the case of Nelson, BC. Can J Rural Med 2013;18:56-61.

33. Rourke JT, Kennard M. Emergency patient transfers from rural hospitals: a regional study. CJEM 2001;3:296-301.

34. Nelson BP, Melnick ER, Li J. Portable ultrasound for remote environments, Part I: feasibility of field deployment. J Emerg Med 2011;40:190-7.

35. Banque de données ministérielles du système d'information du Registre des traumatismes du Québec (BDM-SIRTQ). http://www. ramq.gouv.qc.ca/fr/donnees-statistiques/sur-demande/donneesmsss/Pages/bdm-sirtq.aspx\# (accessed 19 Nov 2014).

36. Hasson F, Keeney S, McKenna H. Research guidelines for the Delphi survey technique. J Adv Nurs 2000;32:1008-15.

37. Keeney S, Hasson F, McKenna HP. A critical review of the Delphi technique as a research methodology for nursing. Int J Nurs Stud 2001;38:195-200.

38. Beattie E, Mackway-Jones K. A Delphi study to identify performance indicators for emergency medicine. Emerg Med J. 2004;21:47-50.

39. Wakai A, O'Sullivan R, Staunton P, et al. Development of key performance indicators for emergency departments in Ireland using an electronic modified-Delphi consensus approach. Eur J Emerg Med 2013;20:109-14.

40. McGinnis PQ, Wainwright SF, Hack LM, et al. Use of a Delphi panel to establish consensus for recommended uses of selected balance assessment approaches. Physiother Theory Pract 2010;26: 358-73.

41. Patton MQ. Qualitative evaluation methods. Beverly Hills, CA, Sage Publications Inc., 1980.

42. Delbecq AL, Van de Ven AH, Gustafson DH. The Delphi technique. Green Briar Press, Ed. In: Group techniques for program planning: a guide to nominal group and Delphi processes. Glenview, IL, Scott, Foresman and Co, 1975:83-107.

43. Guba EG, Lincoln YS. Fourth generation evaluation. Newbury Park, CA, USA: Sage, 1989.

44. Lincoln YS. Fourth generation evaluation, the paradigm revolution and health promotion. Can J Public Health 1992;83(Suppl 1):S6-10.

45. Lincoln YS. Sympathetic connections between qualitative methods and health research. Qual Health Res 1992;2:375-91.

46. Baker SP, O'Neill B, Haddon W Jr, et al. The injury severity score: a method for describing patients with multiple injuries and evaluating emergency care. J Trauma 1974;14:187-96.

47. Garwe T, Cowan LD, Neas BR, et al. Directness of transport of major trauma patients to a level I trauma center: a propensityadjusted survival analysis of the impact on short-term mortality. J Trauma 2011;70:1118-27.

48. D'Agostino RB Jr. Propensity score methods for bias reduction in the comparison of a treatment to a non-randomized control group. Stat Med 1998;17:2265-81.

49. Svenson J. Trauma systems and timing of patient transfer: are we improving? Am J Emerg Med 2008;26:465-8. 